\title{
REVIEW
}

\section{The clinical landscape for SMA in a new therapeutic era}

\author{
K Talbot ${ }^{1,4}$ and EF Tizzano ${ }^{2,3,4}$
}

Despite significant advances in basic research, the treatment of degenerative diseases of the nervous system remains one of the greatest challenges for translational medicine. The childhood onset motor neuron disorder spinal muscular atrophy (SMA) has been viewed as one of the more tractable targets for molecular therapy due to a detailed understanding of the molecular genetic basis of the disease. In SMA, inactivating mutations in the SMN1 gene can be partially compensated for by limited expression of SMN protein from a variable number of copies of the SMN2 gene, which provides both a molecular explanation for phenotypic severity and a target for therapy. The advent of the first tailored molecular therapy for SMA, based on modulating the splicing behaviour of the SMN2 gene provides, for the first time, a treatment which alters the natural history of motor neuron degeneration. Here we consider how this will change the landscape for diagnosis, clinical management and future therapeutic trials in SMA, as well as the implications for the molecular therapy of other neurological diseases.

Gene Therapy (2017) 24, 529-533; doi:10.1038/gt.2017.52

\section{INTRODUCTION}

Broadly defined, neurodegeneration is the progressive loss of specific populations of neurons and the dissolution of integrative networks in the central nervous system and its connections, leading to loss of function and, in many cases, premature mortality. A large number of biological processes and complex clinical phenotypes, including the major types of dementia, make neurodegenerative diseases collectively one of the key public health challenges for both developed and developing societies. Despite a dramatic increase in our understanding of the biology of neurodegeneration, the growing number of high profile clinical trial failures, notably in Alzheimer's disease (AD), but also in degenerative motor neuron diseases such as amyotrophic lateral sclerosis (ALS), is a constant reminder of the difficulties in translating these discoveries into clinically meaningful advances in therapy. ${ }^{1}$ Spinal muscular atrophy (SMA) is not a classical neurodegenerative disease, and has a neurodevelopmental dimension to its pathophysiology. However, it does share a defining neurobiological feature with age-related system degenerations like ALS, in that functional synaptic connections are disrupted and specific nerve cells populations are progressively lost, although the order of these events is yet to be definitively established. In SMA, which typically presents in infancy, there is a profound and catastrophic loss of connectivity between the neuronal endplate and muscle, and death of motor neurons. As a consequence, the dominant clinical feature is muscle weakness, with a spectrum of severity ranging from extremely compromised neonates to late onset, minimally affected, adults (Table 1 and Figure 1).

It is a remarkable fact that the first targeted molecular therapy, which significantly disrupts this neuropathological process, has been achieved at a time when scientists are still uncertain, or at least divided in their opinion, about which of the several functions of the causative gene product is relevant for spinal motor neuron survival. ${ }^{2,3}$ Despite this gap in our knowledge, systematic research over the last two decades has gradually elucidated the precise molecular genetic mechanisms, whereby mutations in the $S M N$ gene give rise to SMA, and has laid the foundations for therapy. The primary determinant of the disease is the deletion or mutation of SMN1, but the clinical phenotype is modified by a paralogue, SMN2, present in all patients at a variable number of copies. ${ }^{4-7}$ As a generalisation, a higher number of copies of SMN2, which produces a small amount of full-length SMN protein, is associated with a less severe phenotype (Table 1 and Figure 1). However, exceptions and discordant cases have been reported and predictions about prognosis in SMA patients should also take into account the age of onset and motor milestones achieved, in addition to SMN2 copy number.

The SMN protein is required for the viability of all eukaryotic cells through its canonical function as a co-factor in the assembly of the spliceosomal complex of proteins. ${ }^{8}$ Homozygous loss of the normal SMN1 gene would normally be fatal, but for a duplication event $\sim 5$ million years ago, leading to creation of the SMN2 gene, which is unique to humans. The small amounts of full-length SMN protein produced from SMN2 are apparently sufficient for the health of most somatic cells with the exception of spinal motor neurons. Pre-clinical models predict that a relatively modest increase in full-length SMN protein would be enough to rescue motor neuron degeneration, making modulation of SMN2 splicing a prime therapeutic target.

Spinal muscular atrophy is now in the vanguard of a new era of neurological therapeutics because of the demonstration that the splice-switching antisense oligonucleotide nusinersen has a clinically meaningful effect, with most trial participants surviving at 2 years after treatment was commenced, past a point when

\footnotetext{
${ }^{1}$ Nuffield Department of Clinical Neurosciences, University of Oxford, John Radcliffe Hospital, Oxford, UK; ${ }^{2}$ Department of Clinical and Molecular Genetics, Rare Diseases Unit, Hospital Valle Hebron, Barcelona, Spain and ${ }^{3}$ CIBERER, Barcelona, Spain. Correspondence: Professor K Talbot, Nuffield Department of Clinical Neurosciences, University of Oxford,

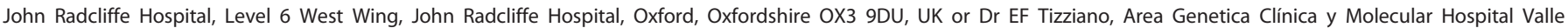
HebronPso. Vall d' Hebron, Barcelona 119-129 08035, Spain.

E-mail: kevin.talbot@ndcn.ox.ac.uk or etizzano@vhebron.net

${ }^{4}$ These authors contributed equally to this work.

Received 3 March 2017; revised 5 June 2017; accepted 12 June 2017; accepted article preview online 23 June 2017; advance online publication, 20 July 2017
} 
Table 1. SMA clinical classification according to onset, achieved milestones, evolution and SMN2 genotype based on experience and previous reports ${ }^{29-32}$

\begin{tabular}{|c|c|c|c|c|}
\hline SMA type & Onset & Milestones achieved & Evolution / natural history & $\begin{array}{c}\text { Typical SMN2 copy } \\
\text { number }\end{array}$ \\
\hline $\begin{array}{l}1 \mathrm{~A} \text { (also referred } \\
\text { as type } 0 \text { ) }\end{array}$ & Prenatal & None & Death in weeks, contractures, cardiopathy & 1 \\
\hline $1 C$ & $>3 M$ & Cephalic control & $\begin{array}{l}\text { Feeding and respiratory problems. Plateau in first two } \\
\text { years }\end{array}$ & 3 \\
\hline $3 a$ & $\begin{array}{l}\text { Between } 18 \text { and } \\
36 \text { months }\end{array}$ & Walking unaided & $\begin{array}{l}\text { Scoliosis } \\
\text { Early loss of ambulation } \\
\text { Normal lifespan }\end{array}$ & 3 \\
\hline $3 b$ & $>3$ years & Walking unaided & $\begin{array}{l}\text { Later loss of ambulation } \\
\text { Normal lifespan }\end{array}$ & $3-4$ \\
\hline 4 & $\begin{array}{l}\text { Second or third decade } \\
\text { of life }\end{array}$ & Walking unaided & $\begin{array}{l}\text { Ambulant until late in life } \\
\text { Normal lifespan }\end{array}$ & 4 \\
\hline
\end{tabular}
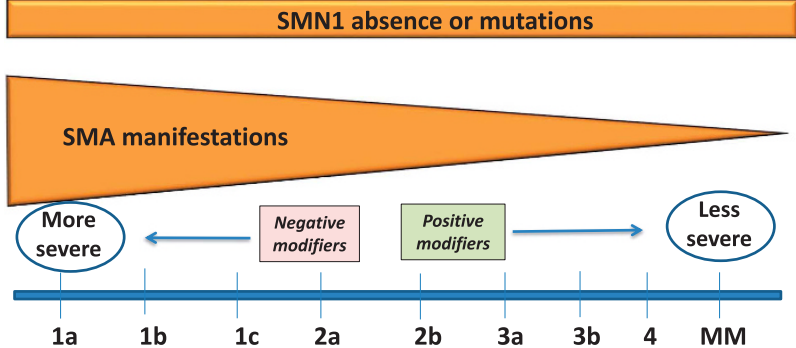

SMN2 copies / Amount of SMN protein

Figure 1. A continuous spectrum of phenotypes in SMA. Despite genetic confirmation of SMN1 absence or mutations in all patients, SMA presentation ranges from very compromised neonates (type $1 \mathrm{~A})$ to adults with minimal manifestations (MM) depending on the number of SMN2 copies and full-length protein produced by each patient and modulated by negative or positive modifiers that influence the final phenotype.

the natural history of untreated severe SMA would have resulted in death or dependence on invasive ventilation. ${ }^{9}$ Moreover, an improvement in motor function was evident, with most of the cases reaching the ceiling of the outcome scale (CHOP INTEND), which has been established to evaluate natural history in type 1 patients. $^{10}$ The detection of improved neurophysiological measures is also an extremely encouraging sign that neuromuscular functional reserve is present, and can be recruited to maintain function, even as the disease is in evolution. Nusinersen or Spinraza (Biogen, Boston, MA, USA) (previously ISIS-SMN $\mathrm{Rx}_{\mathrm{x}}$ and also known as IONIS 396443) is a 2'-O-methoxyethyl (2'MOE) modified antisense oligonucleotide designed to bind to the SMN2 pre-mRNA matching an intronic splicing silencer in intron 7. As a consequence, the negative splicing factors hnRNP $A 1$ and $A 2$ are displaced and unable to interact with the SMN2 pre-mRNA. This promotes the inclusion of exon 7 by an U1snRNP, allowing its recognition in the splicing process and resulting in the production of full-length RNA and a complete functional protein $^{11}$ (Figure 2).

\section{THE CHANGING CLINICAL COURSE OF SMA IN THE THERAPEUTIC ERA}

Due to advances in nutritional and respiratory care, physiotherapy and enablement strategies to maintain independent living, the prognosis of SMA has been changing over the last few decades. ${ }^{12}$ The outlook for even the most severely affected children has improved in terms of crude survival, albeit with severe disability. However, there is no evidence that such management strategies alter the basic neuropathological process and neuromuscular function, and the effects are necessarily very limited in modifying the motor milestones and natural history of the disease.

The most dramatic immediate consequence of the step change in therapy represented by nusinersen is that SMA Type 1 children will achieve motor milestones normally associated with less severe types of SMA (for example, sitting, standing) and are likely to survive in large numbers beyond the point where they would have previously succumbed to respiratory failure without invasive ventilation. This will effectively increase the prevalent SMA population. Depending on how early specific treatment can be instituted, the clinical course of SMA will be altered, but with many more children going into adolescence and adulthood with significant care and enablement needs. Together with the increased resources required to deliver intrathecal therapy and monitor its effects, managing this increased population of SMA patients will require a scaling up in resources for paediatric, and ultimately adult, neuromuscular multidisciplinary teams.

\section{THE IMPACT OF MUTATION SCREENING}

The advent of a therapy that can change the clinical course of SMA, coupled with the fact that all affected individuals will have some degree of irreversible motor neuron loss at diagnosis, argues in favour of treatment being given as early as possible. If, as can be expected, this advance in therapy removes any resistance to mass newborn screening, the next logical direction for treatment would therefore be to identify pre-symptomatic cases and institute therapy at or soon after birth. Newborn screening will detect the majority of SMA cases given that $95 \%$ of SMA patients show absence of exon 7 of the SMN1 gene and it has been demonstrated to be technically feasible. ${ }^{13}$ Subsequent testing for SMN2 copy number may also allow prediction of the phenotype in the presence of one, two or four SMN2 copies. ${ }^{14}$ However, prediction of the ultimate clinical phenotype in cases with three SMN2 copies is at present less certain. 
a

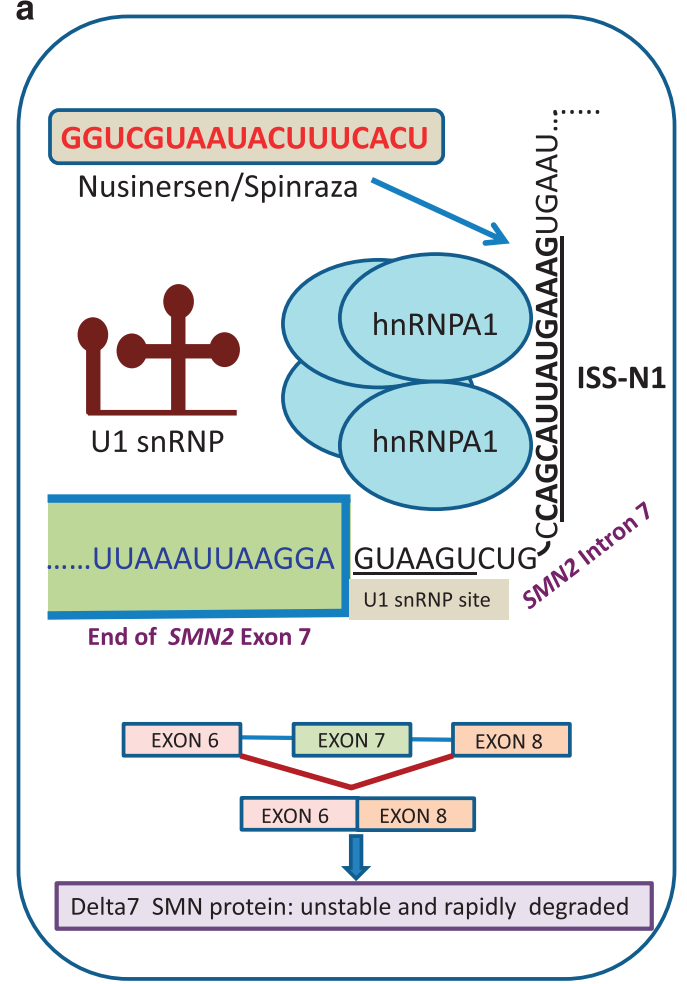

b

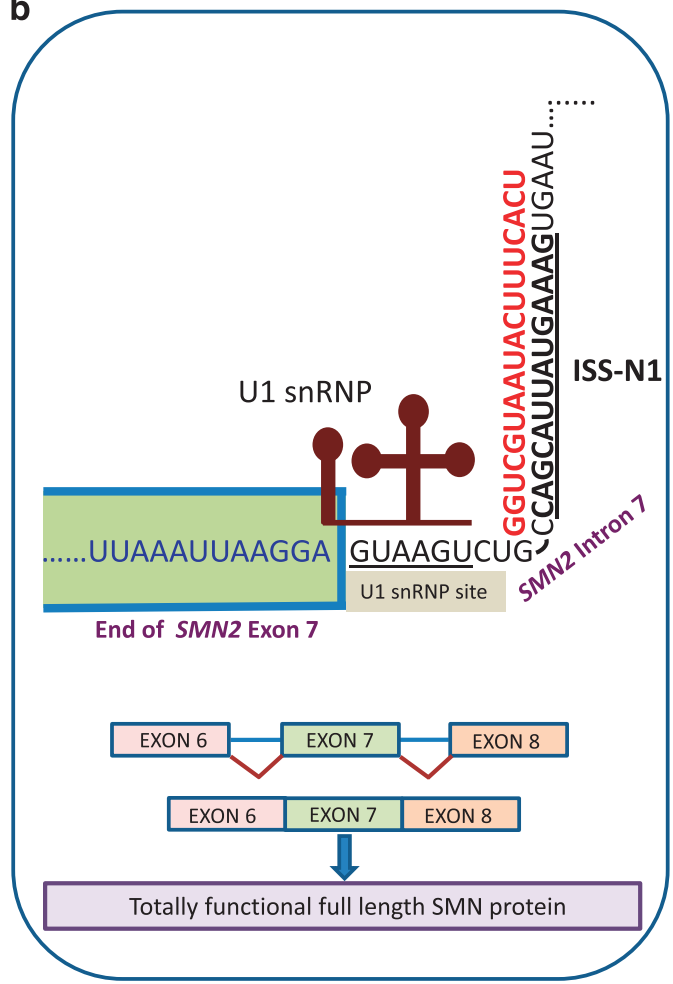

Figure 2. Schematic representation of the mechanism of action of nusinersen (Spinraza). (a) SMN2 intron 7 contains an intronic splicing silencer (termed ISS-N1) with binding sites for negative splicing factors (NSFs), hnRNPA1 and 2. Binding of these NSFs to intron 7 pre-mRNA precludes the interaction of U1 snRNP1 with its specific site at the beginning of the intron and therefore the recognition of exon 7 during the splicing process. (b) The antisense oligonucleotide nusinersen blocks the ISS-N1 site preventing the binding of the NSFs, and allowing U1 snRNP1 to recognise exon 7 of the SMN2 pre-mRNA. As a result, exon 7 is included in the SMN2 mature RNA, and is translated into full-length SMN protein. Based on a bidimensional model by Singh et al. ${ }^{11}$

Mass post-natal screening, using methods that will pick up carriers as well as homozygously deleted SMN1 cases would have long-term consequences, in that all children will grow up knowing their carrier status. It is impossible to predict how this will affect reproductive planning, but it is entirely possible that the incidence of SMA in advanced health care systems will in the long-term shrink to a very low level if the majority of couples in which both are carriers choose termination or pre-implantation genetic diagnosis. Carrier screening in antenatal clinics would have a similar outcome.

\section{POTENTIAL LIMITATIONS OF SMN AUGMENTATION THERAPIES}

Despite the clinically dramatic effect of nusinersen on motor function in SMA, some major issues remain unresolved.

(i) Timing of SMN replacement

In the nusinersen study, there was a median delay of $\sim 100$ days from symptom onset to treatment. Even though this time may be reduced in the clinical context where the drug is freely available, it is inevitable in a disease, which develops sub-acutely in the case of Type I SMA, and over months in milder forms, that there will be delayed diagnosis and irreversible loss of motor function by the time of diagnosis. Natural history studies of SMA are consistent with there being two phases of progression, an early phase of motor neuron loss and a later, slower attrition of motor units, which could have a different mechanistic basis. In type 1 SMA patients, observations of a marked and early loss of MNs with immature morphology of the remaining motor units has been described, and might favour a developmental aspect to SMA pathogenesis, on the background of low levels of SMN during embryogenesis, although this remains unclear. ${ }^{15}$

Pre-clinical studies in mouse models of SMA, however, suggest a discrete time-window in neuromuscular development when increasing SMN levels is effective. Whether any therapeutic effect will be seen when ASO therapy is administered during the later phase of slow decline is unclear at this time. The therapeutic window is also dictated by the rapidity of disease onset. Type 1 infants usually present with rapid evolution of symptoms and are very fragile, with the potential for severe complications, whereas chronic type 2 and 3 cases have a more insidious onset and may experience long periods of stability. ${ }^{16,17}$ Furthermore, the temporal requirements for SMN protein in other non-neuronal tissues are even less well understood.

The devastating nature of clinical progression in untreated Type 1 SMA has allowed the therapeutic effect of nusinersen to be detected in an open label and relatively small-scale study. Assessing the effect of treatment initiated in the chronic phase of disease will be more challenging. Since nusinersen has recently been approved by the FDA and by the EMA for all types of SMA, further formal clinical trials in older children and adults are unlikely, though it may be possible to collect the relevant data from a prospectively monitored cohort of treated individuals using historical controls as a comparator.

\section{(ii) Delivery across the blood-brain barrier}

There is no practical alternative to periodic intrathecal delivery of nusinersen at the present time. Although pharmacokinetic studies indicate a prolonged CSF drug half-life of up to 4-6 months after initial clearance, the current dosing regimen requires treatment every 3-4 months and the intrathecal route therefore represents 
an obvious barrier to the free availability of this treatment in all health care settings due to the extra cost and facilities required. ${ }^{18}$ The intrathecal route will also act as an impediment to treatment in older SMA patients, who have often had spinal surgery to correct or prevent scoliosis. This is likely to require image guided lumbar puncture in most cases, or may be impractical in many individuals.

\section{(iii) CNS versus systemic delivery}

A major issue with intrathecal delivery is the concern that for complete rescue of the disease phenotype SMN upregulation may be required in other tissues, especially at the neuromuscular junctions and in muscle. ${ }^{19,20}$ Mouse studies have demonstrated that systemic delivery leading to peripheral SMN restoration in the absence of CNS expression preserves motor neurons, suggesting SMA is not a cell-autonomous defect of motor neurons, at least in SMA mice. ${ }^{21}$ Perhaps, of most concern is the effect of very low levels of SMN in peripheral tissues and other CNS regions, if SMN levels in the spinal motor neurons are restored in isolation. There is no natural history data available to predict whether, in SMA patients with low SMN2 copy number, low SMN in other organ systems might reveal a hitherto unsuspected systemic vulnerability for example in the cardiovascular, gastrointestinal or immune systems. ${ }^{22}$ Even though patients with type 1 SMA who have been on invasive respiratory ventilation for years have not been reported to develop problems in other organ systems, careful monitoring by multispeciality teams will be mandatory in the new therapeutic era to fully understand the effects of living with low SMN levels in the periphery into adolescence and beyond.

\section{THE FUTURE OF SMA THERAPY}

The children treated in the recent nusinersen trial showed acceptable tolerance to the intrathecal procedure and the vast majority of serious adverse events reported were disease related. ${ }^{23}$ However, it is important to note that these children showed progress but did not achieve completely normal motor function in the timescale reported in the trial, and face an uncertain future as they grow and develop from a baseline of established neuromuscular weakness. Therefore, despite the promise provided by nusinersen, it should be seen as the first step in a transformative environment for SMA therapy. There is still much progress to be made and a number of other approaches are under investigation, including modified ASOs to increase cell penetration, oral small molecule approaches aimed at increasing SMN levels, and virally delivered gene replacement therapy (a list of ongoing clinical trials in SMA is available at https://www.clinicaltrials.gov/). It will be difficult to recruit any drug naïve patients in which these agents can be tried, making it challenging to identify the clinical effectiveness of newer agents, except perhaps as adjunctive therapy. Clinical trial methodology in SMA will have to accommodate this complexity, as it will not be ethically acceptable to prevent subjects in new trials taking an established therapy, if available. Trials and protocols of combinatorial therapies aiming for synergies and complementation are envisaged.

It remains possible that SMN restoration might not be a sufficient therapy for all patients with SMA, either because it functions in a time-dependent window in early development and will never prevent the slow progressive decline seen in older children and adults, or because the typical insidious clinical presentation of milder forms of SMA does not allow early treatment in the absence of post-natal screening. For these reasons, a whole range of other, non-SMN, pathways are under investigation, including those based on modifiers of the pathobiology and phenotype. ${ }^{24,25}$ The future of SMA therapy covering the whole period from infancy to late life may well require a range of therapies in combination. Even if SMA can be treated preclinically, it is well established that in the most severely affected children, the pathological process begins in utero. ${ }^{15}$ Even with the best approaches to SMN replacement, there may be late effects in neuromuscular weakness due to a reduced functional reserve.

\section{WIDER IMPLICATIONS FOR THE TREATMENT OF NEUROLOGICAL DISEASES}

The demonstration of clinical efficacy of nusinersen in SMA represents an important proof-of-principle that antisense oligonucleotide therapy is a viable therapeutic approach in the nervous system. As such, it has wider implications for genetically determined neurodegenerative diseases, including forms of ALS, triplet repeat diseases like Huntington's disease, myotonic dystrophy and others where ASOs could be developed to block the expression of a toxic DNA fragment such as a triplet repeat or the hexanucleotide repeat in the C9orf72 gene responsible for $10 \%$ of ALS cases and a similar number of cases of frontotemporal dementia. In addition, there is preliminary evidence that SMN may have neuroprotective effects in ALS, rescuing motor neuron death in a mouse model, suggesting that a clinical trial of nusinersen in ALS may be worth considering. ${ }^{26}$ In other neuromuscular disorders such as Duchenne muscular dystrophy, antisense therapy is also under evaluation. ${ }^{27}$ Other technologically advanced strategies such as genome editing with CRISPR/Cas9 are under pre-clinical investigation. ${ }^{28}$ The history of SMA is a key example of how knowledge of the basic molecular genetic mechanism of a disease, coupled with detailed natural history studies, evidence-based management and standards of care combine to facilitate the translation of basic science into lifetransforming therapy.

\section{CONFLICT OF INTEREST}

The authors declare no conflict of interest.

\section{ACKNOWLEDGEMENTS}

The authors acknowledge the useful discussions with colleagues and patients which have informed the opinions expressed in this article. EFT is a recipient of funding from SMA Europe and Daniel Bravo for SMA. KT receives funding from the SMA Trust and the Motor Neuron Disease Association. EFT has received grant support to conduct clinical trials on SMA from lonis/Biogen.

\section{REFERENCES}

1 Cudkowicz ME, van den Berg LH, Shefner JM, Mitsumoto H, Mora JS, Ludolph A et al. Dexpramipexole versus placebo for patients with amyotrophic lateral sclerosis (EMPOWER): a randomised, double-blind, phase 3 trial. Lancet Neurol 2013; 12: 1059-1067

2 Singh RN, Howell MD, Ottesen EW, Singh NN. Diverse role of survival motor neuron protein. Biochim Biophys Acta 2017; 1860: 299-315.

3 Burghes $\mathrm{AH}$, Beattie CE. Spinal muscular atrophy: why do low levels of survival motor neuron protein make motor neurons sick? Nat Rev Neurosci 2009; 10: 597-609.

4 Lefebvre S, Burglen L, Reboullet S, Clermont O, Burlet $P$, Viollet L et al. Identification and characterization of a spinal muscular atrophy- determining gene [see comments]. Cell 1995; 80: 155-165.

5 Alias L, Bernal S, Fuentes-Prior P, Barcelo MJ, Also E, Martinez-Hernandez R et al. Mutation update of spinal muscular atrophy in Spain: molecular characterization of 745 unrelated patients and identification of four novel mutations in the SMN1 gene. Hum Genet 2009; 125: 29-39.

6 Wirth B. An update of the mutation spectrum of the survival motor neuron gene (SMN1) in autosomal recessive spinal muscular atrophy (SMA). Hum Mutat 2000; 15: 228-237.

7 Feldkotter M, Schwarzer V, Wirth R, Wienker TF, Wirth B. Quantitative analyses of $s m n 1$ and smn2 based on real-time lightcycler pcr: fast and highly reliable carrier testing and prediction of severity of spinal muscular atrophy. Am J Hum Genet 2002; 70: 358-368.

8 Gubitz AK, Feng W, Dreyfuss G. The SMN complex. Exp Cell Res 2004; 296: 51-56. 
9 Finkel RS, Chiriboga CA, Vajsar J, Day JW, Montes J, De Vivo DC et al. Treatment of infantile-onset spinal muscular atrophy with nusinersen: a phase 2, open-label, dose-escalation study. Lancet 2016; 388: 3017-3026.

10 Glanzman AM, Mazzone E, Main M, Pelliccioni M, Wood J, Swoboda KJ et al. The children's hospital of philadelphia infant test of neuromuscular disorders (CHOP INTEND): test development and reliability. Neuromuscul Disord 2010; 20: 155-161.

11 Singh NN, Lee BM, DiDonato CJ, Singh RN. Mechanistic principles of antisense targets for the treatment of spinal muscular atrophy. Future Med Chem 2015; 7: 1793-1808.

12 Wang $\mathrm{CH}$, Finkel RS, Bertini ES, Schroth M, Simonds A, Wong B et al. Consensus statement for standard of care in spinal muscular atrophy. J Child Neurol 2007; 22: 1027-1049.

13 Phan HC, Taylor JL, Hannon H, Howell R. Newborn screening for spinal muscular atrophy: Anticipating an imminent need. Semin Perinatol 2015; 39: 217-229.

14 Cusco I, Barcelo MJ, Rojas-Garcia R, Illa I, Gamez J, Cervera C et al. SMN2 copy number predicts acute or chronic spinal muscular atrophy but does not account for intrafamilial variability in siblings. J Neurol 2006; 253: 21-25.

15 Perez Garcia M, Kong L, Sumner C, Tizzano E. Developmental Aspects and Pathological findigins in Spinal Muscular Atrophy. In: Sumner CJ, Paushkin S, Ko CPeditors. Spinal Muscular Atrophy: Disease Mechanisms and Therapy. Elsevier/ Academic Press: Amsterdam, The Netherlands, 2017, p xxxii 474 pages.

16 Kaufmann P, McDermott MP, Darras BT, Finkel RS, Sproule DM, Kang PB et al. Prospective cohort study of spinal muscular atrophy types 2 and 3. Neurology 2012; 79: 1889-1897.

17 Mercuri E, Finkel R, Montes J, Mazzone ES, Sormani MP, Main M et al. Patterns of disease progression in type 2 and 3 SMA: Implications for clinical trials. Neuromuscul Disord 2016; 26: 126-131.

18 Chiriboga CA, Swoboda KJ, Darras BT, lannaccone ST, Montes J, De Vivo DC et al. Results from a phase 1 study of nusinersen (ISIS-SMN(Rx)) in children with spinal muscular atrophy. Neurology 2016; 86: 890-897.

19 Hamilton G, Gillingwater TH. Spinal muscular atrophy: going beyond the motor neuron. Trends Mol Med 2013; 19: 40-50.

20 Harding BN, Kariya S, Monani UR, Chung WK, Benton M, Yum SW et al. Spectrum of neuropathophysiology in spinal muscular atrophy type I. J Neuropathol Exp Neurol 2015; 74: 15-24.

21 Hua Y, Liu YH, Sahashi K, Rigo F, Bennett CF, Krainer AR. Motor neuron cellnonautonomous rescue of spinal muscular atrophy phenotypes in mild and severe transgenic mouse models. Genes Dev 2015; 29: 288-297.

22 Hua Y, Sahashi K, Rigo F, Hung G, Horev G, Bennett CF et al. Peripheral SMN restoration is essential for long-term rescue of a severe spinal muscular atrophy mouse model. Nature 2011; 478: 123-126.

23 Hache M, Swoboda KJ, Sethna N, Farrow-Gillespie A, Khandji A, Xia S et al. Intrathecal injections in children with spinal muscular atrophy: nusinersen clinical trial experience. J Child Neurol 2016; 31: 899-906.
24 Bertini E, Dessaud E, Mercuri E, Muntoni F, Kirschner J, Reid C et al. Safety and efficacy of olesoxime in patients with type 2 or non-ambulatory type 3 spinal muscular atrophy: a randomised, double-blind, placebo-controlled phase 2 trial. Lancet Neurol 2017; 16: 513-522.

25 Riessland M, Kaczmarek A, Schneider S, Swoboda KJ, Lohr H, Bradler C et al. Neurocalcin delta suppression protects against spinal muscular atrophy in humans and across species by restoring impaired endocytosis. Am J Hum Genet 2017; 100: 297-315.

26 Turner BJ, Alfazema N, Sheean RK, Sleigh JN, Davies KE, Horne MK et al. Overexpression of survival motor neuron improves neuromuscular function and motor neuron survival in mutant SOD1 mice. Neurobiol Aging 2014; 35: 906-915.

27 Niks EH, Aartsma-Rus A. Exon skipping: a first in class strategy for Duchenne muscular dystrophy. Expert Opin Biol Ther 2017; 17: 225-236.

28 Bengtsson NE, Hall JK, Odom GL, Phelps MP, Andrus CR, Hawkins RD et al. Muscle-specific CRISPR/Cas9 dystrophin gene editing ameliorates pathophysiology in a mouse model for Duchenne muscular dystrophy. Nat Commun 2017; 8: 14454.

29 Bertini E, Burghes A, Bushby K, Estournet-Mathiaud B, Finkel RS, Hughes RA et al.. 134th ENMC International Workshop: Outcome Measures and Treatment of Spinal Muscular Atrophy, 11-13 February 2005, Naarden, The Netherlands. Neuromuscul Disord 2005; 15: 802-816.

30 Rudnik-Schoneborn S, Forkert R, Hahnen E, Wirth B, Zerres K. Clinical spectrum and diagnostic criteria of infantile spinal muscular atrophy: further delineation on the basis of SMN gene deletion findings. Neuropediatrics 1996 ; 27: 8-15.

31 Rudnik-Schoneborn S, Hausmanowa-Petrusewicz I, Borkowska J, Zerres K The predictive value of achieved motor milestones assessed in 441 patients with infantile spinal muscular atrophy types II and III. Eur Neurol 1996; 45: 174-181.

32 Finkel RS, McDermott MP, Kaufmann P, Darras BT, Chung WK, Sproule DM et al. Observational study of spinal muscular atrophy type I and implications for clinical trials. Neurology 2014; 83: 810-817.

(c) (i) $\odot$ This work is licensed under a Creative Commons AttributionCy No ND NonCommercial-NoDerivs 4.0 International License. The images or other third party material in this article are included in the article's Creative Commons license, unless indicated otherwise in the credit line; if the material is not included under the Creative Commons license, users will need to obtain permission from the license holder to reproduce the material. To view a copy of this license, visit http:// creativecommons.org/licenses/by-nc-nd/4.0/

(c) The Author(s) 2017 